\title{
Die Auslegung künftiger EU-Strafrechtskompetenzen nach dem Lissabon-Urteil des Bundesverfassungsgerichts
}

\author{
Von Wiss. Mit. Frank Zimmermann, München*
}

Der Vertrag von Lissabon bringt gerade im Bereich des Straf- und Strafprozessrechts erhebliche Kompetenzverschiebungen mit sich. Die künftigen Befugnisse der EU auf diesem Gebiet sind deshalb auch für Studierende des Schwerpunktbereichs »Europäisches Strafrecht « von großem Interesse. Mittlerweile hat sich das BVerfG zu dem Vertragswerk geäußert ${ }^{1}$ und sich erneut ${ }^{2}$ damit befasst, welche Grenzen das Grundgesetz dem Prozess der Europäischen Integration setzt. Die Auslegung der neuen Kompetenznormen und die Konsequenzen, welche das Urteil hierfür entfaltet, stehen im Mittelpunkt dieses Beitrags.

\section{Einleitung}

Der im Dezember 2007 von den Staats- und Regierungschefs der EU unterzeichnete Vertrag von Lissabon soll die EU grundlegend reformieren ${ }^{3}$. Eine zentrale Rolle spielen dabei auch die Zuständigkeiten auf dem Gebiet des Strafrechts, die künftig in größerem Umfang als bisher auf europäischer Ebene ausgeübt werden sollen. Zunächst müssen jedoch alle 27 Mitgliedstaaten den Vertrag ratifizieren. Zu diesem Zweck haben Bundestag und Bundesrat ein Zustimmungsgesetz gem. Art. 23 I 2 GG erlassen, das von einem Gesetz zur Änderung des Grundgesetzes und einem Begleitgesetz umrahmt wird. Gegen diese Gesetzgebung wurden allerdings mehrere Verfassungsbeschwerden erhoben, so dass Bundespräsident Köhler mit dem Unterschreiben der Ratifikationsurkunde wartete. Das Urteil aus Karlsruhe wurde deshalb mit Spannung erwartet. Ohne zuviel vorwegzunehmen darf es nicht zuletzt aus Sicht des Europäischen Strafrechts - als ein echtes Ausrufezeichen bezeichnet werden.

\section{Die Bedeutung des Vertrags von Lissabon für das Strafrecht}

Um die Tragweite des Urteils erfassen zu können, soll zunächst erörtert werden, inwiefern der Lissabon-Vertrag die strafrechtliche Kompetenzlage auf europäischer Ebene modifiziert. Dabei sollen auch Auslegungsprobleme bzgl. der neuen Vorschriften zur Sprache kommen. Soweit das Urteil des BVerfg hierfür relevant ist, werden sie später (unten IV.) wieder aufgegriffen.

1. Strukturelle Änderungen und ihre Folgen für das Strafrecht Der Vertrag von Lissabon scheint zunächst keine große Neuerung zu bringen: Der EG-Vertrag firmiert künftig lediglich als »Vertrag über die Arbeitsweise der Europäischen Union« (AEUV) ${ }^{4}$. An dem Prinzip der begrenzten Einzelermächtigung wird festgehalten (Art. 5 I 1, II EUV nF), so dass die Union weiterhin nur tätig werden kann, wenn ihr hierfür eine konkrete Befugnis zugewiesen wurde $^{5}$. Zudem bleibt der Subsidiaritätsgrundsatz, wonach die EU nur handeln darf, wenn eine Maßnahme nicht genauso gut von den Mitgliedstaaten durchgeführt werden könnte ${ }^{6}$, gem. Art. 5 I 2, III EUV nF ein Leitprinzip des EU-Rechts.

Bei näherem Hinsehen sind die Änderungen durch die Vertragsreform indes kaum zu unterschätzen ${ }^{7}$. Sie sind zumeist Ausdruck der Zielsetzung, die Union auch mit derzeit 27 Mitgliedstaaten handlungsfähig zu machen. So löst der Lissabon-Vertrag die Säulenstruktur der Europäischen Union ${ }^{8}$ weitgehend auf, die Polizeiliche und Justitielle Zusammenarbeit in Strafsachen (PJZS, bisher 3. Säule der EU), die intergouvernemental organisiert war, wird in die Supranationalität (bisher EG, 1. Säule der
EU) überführt. Für den Bereich des Strafrechts hat schon diese strukturelle Verschiebung erhebliche Auswirkungen:

Erstens könnte das Straf- und Strafprozessrecht durch Richtlinien (statt Rahmenbeschlüsse) vereinheitlicht werden, für die das »ordentliche Gesetzgebungsverfahren « gilt. Gem. Art. 289 I, 294 AEUV entscheiden dann Rat und Europäisches Parlament (EP) gemeinsam - bei der Rechtssetzung iRd PJZS wurde das Parlament demgegenüber nur angehört (Art. 39 I 1 EUV). Dies führt im Bereich des Strafrechts zu einer überaus wünschenswerten Stärkung der demokratischen Legitimation ${ }^{9}$.

Zweitens genügt gem. Art. 16 III EUV nF bei der Abstimmung über eine solche Richtlinie im Rat grundsätzlich eine qualifizierte Mehrheit ${ }^{10}$, wohingegen Rahmenbeschlüsse gem. Art. 34 II 2 EUV nur einstimmig ergehen konnten. Selbst in Bereichen, in denen weiterhin das Einstimmigkeitsprinzip gilt, kann mittels sog. Brückenklauseln (zB Art. 48 VII EUV nF) zu Mehrheitsentscheidungen übergegangen werden. Erforderlich ist dafür ein einstimmiger Beschluss des Europäischen Rates der Staatsund Regierungschefs; die nationalen Parlamente können einen solchen Beschluss binnen sechs Monaten ablehnen.

Drittens zeigt sich die schwächere Position der Mitgliedstaaten beim Initiativrecht für den Erlass einer strafrechtlichen Richtlinie: Gem. Art. 76 AEUV soll es neben der Kommission einer Gruppe von mindestens einem Viertel der Mitgliedstaaten zustehen; bisher konnte nach Art. 34 II 2 EUV auch ein einzelner Mitgliedstaat eine solche Initiative ergreifen.

Schließlich ist viertens die Umsetzung strafrechtlicher EUVorgaben künftig mittels des Vertragsverletzungsverfahrens (Art. 258 ff. AEUV) überprüfbar. Dies gilt nach Art. 35 EUV für EU-Rahmenbeschlüsse nicht. Eine Sonderregelung trifft deshalb Art. 10 I i. V. m. III des zukünftigen Protokolls Nr. 36 über Übergangsbestimmungen ${ }^{11}$ bzgl. heute schon bestehender Rechtsakte, die iRd PJZS ergangen sind (etwa den Rahmenbeschluss über den Europäischen Haftbefehl ${ }^{12}$ ): Ihre Implementierung kann erst nach einer Übergangsfrist von fünf Jahren ab dem Inkrafttreten des Lissabon-Vertrags durch den EuGH kontrolliert werden.

Abgesehen von diesen generellen Veränderungen bringt namentlich die Ausgestaltung der Kompetenzvorschriften im Einzelnen zahlreiche Umwälzungen mit sich.

* Der Autor ist wissenschaftlicher Mitarbeiter am Lehrstuhl von Prof. Dr. Helmut Satzger (Ludwig-Maximilians-Universität München).

$1 \mathrm{Az} 2 \mathrm{BvE} 2 / 08$.

2 S. bislang etwa BVerfGE 37, 271 (Solange I), BVerfGE 73, 339 (Solange II); BVerfGE 89, 155 (Maastricht), BVerfGE 113, 273 (Europäischer Haftbefehl).

3 Für einen Überblick über die Entstehungsgeschichte und das Ratifikationsverfahren s. Haratsch/Koenig/Pechstein Europarecht, 6. Aufl. 2009, Rdn. 35 a f.

4 Eine konsolidierte Fassung der Verträge findet sich in ABl 2008 Nr. C 115.

5 Ausf. Streinz Europarecht, 9. Aufl. 2008, Rdn. 498; Herdegen Europarecht, 11. Aufl. 2009, $\$ 9$ Rdn. 55.

6 Streinz Europarecht, Rdn. 166; Herdegen Europarecht, $\$ 7$ Rdn. $25 \mathrm{ff}$. 7 S. den Überblick bei Haratsch/Koenig/Pechstein Europarecht, Rdn. $36 \mathrm{ff}$.

8 S. SATZGER Internationales und Europäisches Strafrecht, 3. Aufl. 2009, \$7 Rdn. 31; Herdegen Europarecht, $\$ 5$ Rdn. 1.

9 Sieber ZStW 121 (2009), 1, 57 f.; deutlich zum »Demokratiedefizit« bei Rechtsakten iRd PJZS SCH ÜNEMANN in: ders. (Hrsg.), Ein Gesamtkonzept für die europäische Strafrechtspflege, 2006, $95 \mathrm{ff}$.

10 S. zu Details Art. 16 IV EUV nF (Initiativen der Kommission) und Art. 238 II AEUV (Initiativen eines Viertels der Mitgliedstaaten).

$11 \mathrm{ABl} 2008$ Nr. C 115, $322 \mathrm{ff}$.

$12 \mathrm{ABl} 2002$ Nr. L 190, $1 \mathrm{ff}$. 


\section{Neuerungen bei der Zusammenarbeit in Strafsachen}

a) Normierung des Grundsatzes der gegenseitigen Anerkennung Art. 82 I AEUV führt den Grundsatz der gegenseitigen Anerkennung in Strafsachen, der schon seit dem Europäischen Rat von Tampere als »Eckstein « der PJZS bezeichnet wird, »offiziell « in das europäische Primärrecht ein. Dementsprechend findet sich in Art. 82 I lit. a AEUV eine Kompetenznorm für den Erlass von Rechtsakten, welche die unionsweite Anerkennung gerichtlicher Entscheidungen vereinfachen. In dieselbe Richtung deutet Art. 82 I lit. d AEUV, welcher es der Union erlaubt, Maßnahmen zur Erleichterung der Zusammenarbeit in den Bereichen der Strafverfolgung, des Vollzugs und der Vollstreckung von Entscheidungen zu treffen. Eine grundlegende Neuerung bedeutet all dies freilich nicht, denn bereits auf Grundlage des geltenden Rechts wurden zahlreiche Rahmenbeschlüsse zur Umsetzung des Prinzips der gegenseitigen Anerkennung verabschiedet. Diese wurden bislang auf Art. 29 II, 2. Spiegelstrich i. V. m. Art. 31 I lit. a, b EUV gestützt ${ }^{13}$.

Beispiele: Rahmenbeschluss über den Europäischen Haftbefeh $l^{14}$ (Vollstreckung im EU-Ausland ergangener Haftbefehle), Rahmenbeschluss über die Europäische Beweisanordnung ${ }^{15}$ (Durchführung im Ausland angeordneter Maßnahmen, um Beweise für Strafverfahren zu erheben), Rahmenbeschluss über die Europäische Vollstreckungsanordnung $^{16}$ (Vollstreckung im Ausland verhängter Freiheitsstrafen und Maßregeln)

\section{b) Zusätzliche EU-Kompetenzen zur Angleichung des Strafprozessrechts}

Die gegenseitige Anerkennung justitieller Entscheidungen setzt ein hohes Maß an Vertrauen in die straf(prozess)rechtlichen Systeme der anderen Mitgliedstaaten voraus. Erhebliche Unterschiede zwischen den strafprozessualen Vorschriften stehen diesem Vertrauen entgegen und bilden ein massives Hindernis für die Realisierung des Anerkennungsgrundsatzes. Eine Angleichung des Strafprozessrechts war bisher jedoch allenfalls auf Grundlage des Art. 31 I lit. c EUV möglich, der (reichlich nebulös) von der »Gewährleistung der Vereinbarkeit der jeweils geltenden Vorschriften der Mitgliedstaaten untereinander " sprach $^{17}$. So wurde bis heute auch nur ein Rahmenbeschluss erlassen, welcher gezielt das Strafprozessrecht - konkret die Rechte des Opfers im Strafverfahren - vereinheitlichte ${ }^{18}$. Ein Vorschlag für einen Rahmenbeschluss über Beschuldigtenrechte kam dagegen, nicht zuletzt wegen kompetenzrechtlicher Bedenken, nicht zustande ${ }^{19}$. Deshalb enthält Art. 82 II AEUV eine explizite Befugnis der EU zur Angleichung des Strafprozessrechts:

(1) $(\ldots)$

(2) Soweit dies zur Erleichterung der gegenseitigen Anerkennung gerichtlicher Urteile und Entscheidungen und der polizeilichen und justiziellen Zusammenarbeit in Strafsachen mit grenzüberschreitender Dimension erforderlich ist, können das Europäische Parlament und der Rat gemäß dem ordentlichen Gesetzgebungsverfahren durch Richtlinien Mindestvorschriften festlegen. Bei diesen Mindestvorschriften werden die Unterschiede zwischen den Rechtsordnungen und -traditionen der Mitgliedstaaten berücksichtigt.

Die Vorschriften betreffen Folgendes:

a) die Zulässigkeit von Beweismitteln auf gegenseitiger Basis zwischen den Mitgliedstaaten;

b) die Rechte des Einzelnen im Strafverfahren;

c) die Rechte der Opfer von Straftaten;

d) sonstige spezifische Aspekte des Strafverfahrens, die zuvor vom Rat durch Beschluss bestimmt worden sind; dieser Beschluss wird vom Rat einstimmig nach Zustimmung des Europäischen Parlaments erlassen.

Beachtung verdient insbesondere die in Art. 82 II UA 2 lit.d enthaltene Blankettermächtigung, auch »sonstige spezifische Aspekte des Strafverfahrens « zu vereinheitlichen. Obgleich das Einstimmigkeitserfordernis bei der Beschlussfassung im Rat eine gewisse Hürde darstellt, kann auf dieser Grundlage prinzipiell jede Regelung des Strafprozessrechts europaweit harmonisiert werden-zumindest soweit dies iSd Art. 82 II UA 1 erforderlich ist.

\section{c) Kompetenz zur Schaffung einer Europäischen Staats- anwaltschaft}

Schon seit einigen Jahren wird diskutiert, ob der Schutz der finanziellen Interessen der EG gegen Betrügereien sich nicht effektiver durch eine zentrale Europäische Staatsanwaltschaft bewirken ließe, als im Wege der strafrechtlichen Zusammenarbeit zwischen den Mitgliedstaaten ${ }^{20}$. In Art. 86 I AEUV findet sich nun eine Rechtsgrundlage für die Schaffung einer solchen Einrichtung. Bemerkenswert ist, dass gem. Art. 86 I UA 3 schon eine Gruppe von nur neun Mitgliedstaaten sich im Wege einer verstärkten Zusammenarbeit hierzu entschließen könnte. Der Europäische Staatsanwalt würde vor den nationalen Strafgerichten auftreten, dabei käme zumindest teilweise ein originär europäisches Strafprozessrecht zur Anwendung (vgl. Art. 86 III). Inhaltlich beschränkt Art. 86 I seine Befugnisse auf Delikte zum Nachteil der finanziellen Interessen der EU. Jedoch enthält Art. 86 IV eine weitere Generalklausel, wonach die Zuständigkeit durch einstimmigen Ratsbeschluss auf andere Bereiche der schweren Kriminalität ausgedehnt werden kann.

\section{EU-Kompetenzen im Bereich des materiellen Strafrechts}

Teils weitreichende Veränderungen bringt der Lissabon-Vertrag im Hinblick auf die Kompetenzen der EU im Bereich des materiellen Strafrechts mit sich. Insoweit gilt es zunächst zwischen der Kompetenz zur Rechtssetzung und derjenigen zur Rechtsangleichung (Harmonisierung) zu unterscheiden.

\section{a) Kompetenz zur Setzung supranationalen Strafrechts}

Relativ unstreitig ist, dass bisher weder in der 1. noch in der 3. Säule eine Zuständigkeit der EU-Organe für die Setzung echten europäischen Strafrechts enthalten ist ${ }^{21}$. Die Schaffung eines europäischen Strafgesetzbuchs oder auch nur einzelner supranationaler Straftatbestände ist somit derzeit nicht möglich. Auch Art. 280 IV EGV, der sich noch am ehesten als Grundlage für ein EU-Betrugsstrafrecht heranziehen ließe, enthält in Satz 2 einen Vorbehalt, wonach »die Anwendung des Strafrechts der Mitgliedstaaten und ihre Strafrechtspflege ... unberührt « bleiben ${ }^{22}$. Der Lissabon-Vertrag sieht nun eine Modifikation des Vertragstextes vor, welche zunächst ganz unscheinbar wirkt, aber weitreichende Konsequenzen hat. Die neue Fassung der Vorschrift zur Betrugsbekämpfung (Art.325 AEUV) unterscheidet sich nämlich in einem entscheidenden Punkt von Art. 280 EGV:

(1) Die Union und die Mitgliedstaaten bekämpfen Betrügereien und sonstige gegen die finanziellen Interessen der Union gerichtete rechtswidrige Handlungen mit Maßnahmen nach diesem Artikel, die abschreckend sind und in den Mitgliedstaaten sowie in den Organen, Einrich-

13 Bedenken bzgl. der Kompetenzgrundlage äußert zB KaIAfA-Gbandi ZIS 2006, 521, 527.

14 ABl 2002 Nr. L 190, 1

15 ABl 2008 Nr. L 350, 72

$16 \mathrm{ABl} 2008$ Nr. L 327, 27.

17 In diesem Sinne Streinz/SAtzger EUV/EGV, 2003, Art. 31 EUV Rdn. 9. $18 \mathrm{ABl} 2001 \mathrm{Nr}$. L 82, 1.

19 Der ursprüngliche Vorschlag ist enthalten in KOM (2004) 328 endg. Zu den Gründen des Scheiterns s. Fletcher/Lööf/Gilmore EU Criminal Law and Justice, 2008, $127 \mathrm{ff}$.

20 S. das diesbzgl. Grünbuch der Kommission, KOM (2001) 715 endg.; kritisch SATzGer IntStR, $\$ 9$ Rdn. 23 m. w. N.

21 Амвоs Internationales Strafrecht, 2. Aufl. 2008, $\$ 11$ Rdn. 6 ff.; DANNECKer JURA 2006, 95, 96; Hecker Europäisches Strafrecht, 2. Aufl. 2007, $\$ 4$ Rdn. $90 \mathrm{ff}, 101$; SATzGER IntStR, $\$ 7$ Rdn. $25 \mathrm{ff}$; anders etwa Tiedemann Wirtschaftsstrafrecht BT, 2. Aufl. 2008, Rdn.37; Ders. GA $1998,107,108$

22 Zur kontroversen Auslegung dieser Norm SATzGer IntStR, $\$ 7$ Rdn. 28; DAnnecker JURA 2006, 95, 97; Fromm ZIS 2007, 26. 
tungen und sonstigen Stellen der Union einen effektiven Schutz bewirken.

$$
\text { (...) }
$$

(4) Zur Gewährleistung eines effektiven und gleichwertigen Schutzes in den Mitgliedstaaten sowie in den Organen, Einrichtungen und sonstigen Stellen der Union beschließen das Europäische Parlament und der Rat gemäß dem ordentlichen Gesetzgebungsverfahren nach Anhörung des Rechnungshofs die erforderlichen Maßnahmen zur Verhütung und Bekämpfung von Betrügereien, die sich gegen die finanziellen Interessen der Union richten.

In Art. 325 IV AEUV fehlt der bisher in Art. 280 IV 2 EGV enthaltene Vorbehalt zugunsten des Strafrechts der Mitgliedstaaten. Dies lässt nur einen Schluss zu: Durch Art. 325 IV AEUV wird erstmalig eine EU-Kompetenz zum Erlass strafrechtlicher Verordnungen, dh zur Schaffung supranationaler europäischer Straftatbestände, begründet ${ }^{23}$. Auch wenn Art. 325 IV AEUV lediglich von »Maßnahmen zur Bekämpfung und Verhütung von Betrügereien « spricht, scheint es indes nicht ausgeschlossen, dass nach dem Inkrafttreten des Lissabon-Vertrags weitere »EuropaDelikte ${ }^{24}$ eingeführt werden: Denn mit "Betrügereien « iSd Art. 325 IV AEUV muss nicht unbedingt ein Betrug gem. $\$ 263$ I StGB gemeint sein. Vielmehr könnte man hierunter schon ausgewählte Täuschungshandlungen verstehen, so dass zB auch Fälle von Urkundenfälschung erfasst wären; dadurch darf aber nicht der Unterschied zwischen Abs. 1 und 4 (s. sogleich) eingeebnet werden ${ }^{25}$.

Abgesehen davon ließe sich möglicherweise auch in Art. 325 I AEUV eine deutlich weiter reichende Ermächtigung zur Schaffung europäischer Straftatbestände erblicken: Diese Vorschrift spricht anders als Abs. 4 nicht nur von Maßnahmen gegen Betrügereien, sondern (allgemeiner) auch von Schritten gegen »sonstige gegen die finanziellen Interessen der Union gerichtete rechtswidrige Handlungen«. Hierunter könnten etwa auch die Bestechung von EU-Beamten oder die Veruntreuung von EUGeldern gefasst werden. Welche Schritte die Union zu diesem Zweck einleiten kann, wird aus Art. 325 I zwar nicht hinreichend deutlich. Jedoch ließe sich die Formulierung »Maßnahmen nach diesem Artikel« so verstehen, dass damit auf Abs. 4 verwiesen wird. Die darin normierte Kompetenz zum Erlass strafrechtlicher Verordnungen würde sich dann auch auf den gesamten Anwendungsbereich des Art.325 I (»sonstige rechtswidrige Handlungen«) erstrecken. Überzeugend wäre eine solche Lesart allerdings nicht. Zum einen ergibt die Formulierung »Maßnahmen nach diesem Artikel« nämlich auch anderweitig Sinn: Die grammatikalische Auslegung (Subjekt des Satzes sind auch die Mitgliedstaaten!) zeigt, dass sie sich nicht nur auf Aktivitäten der Union, sondern auch auf nationale Bestrebungen bezieht, wie sie die Abs. 2 und 3 vorschreiben. Zum anderen wäre Art. 325 AEUV ein wahres gesetzessystematisches Kuriosum, wenn die allgemeinere Vorschrift des ersten Absatzes hinsichtlich ihrer Rechtsfolge - der Kompetenz, Maßnahmen im Wege des ordentlichen Gesetzgebungsverfahrens zu ergreifen - auf den eigentlich spezielleren Abs. 4 verweisen würde. Schließlich spricht auch die Entstehungsgeschichte des Art. 325 AEUV gegen ein solches Verständnis: Bis auf die Streichung des zweiten Satzes in Abs. 4 wurde der frühere Art. 280 EGV wortgleich übernommen; auch die Verweisung auf »Maßnahmen nach diesem Artikel « existierte in der Vorgängernorm bereits. Dass ein strafrechtlicher Vorbehalt früher nur in Abs. 4 für notwendig erachtet wurde, zeigt, dass die Vertragsparteien in Abs. 1 keine so weitreichende Ermächtigung sahen. Im Übrigen hätte der Vorbehalt ansonsten auch schlicht umgangen werden können. Aus alledem folgt, dass Art. 325 I AEUV lediglich als programmatische Ansage zu verstehen ist; die eigentliche Kompetenz zum Erlass europäischer Rechtsakte findet sich in Art. 325 IV AEUV $^{26}$.

In jedem Fall aber käme ein europäischer Betrugstatbestand kaum gänzlich ohne AT-Regelungen, zB zu Fragen der Täter- schaft und Teilnahme, der Versuchsstrafbarkeit oder der Schuldfähigkeit, aus, wenn er wirklich das Maß an Rechtsvereinheitlichung herstellen soll, das man sich von ihm verspricht. Sollte die EU von ihrer Kompetenz gem. Art. 325 IV AEUV Gebrauch machen, würde dies deshalb weite Kreise ziehen und die Rechtssetzung könnte kaum nach der Schaffung einer isolierten Strafvorschrift Halt machen.

\section{b) Kompetenz zur Harmonisierung des nationalen Strafrechts}

\section{aa) Besonders schwere Kriminalität}

Anders als für die Schaffung supranationalen Strafrechts existieren für eine Angleichung der mitgliedstaatlichen Strafvorschriften schon bisher Kompetenzen der EU. Primär finden sie sich in Art. 29 II, 3. Spiegelstrich i. V. m. Art. 31 I lit. e EUV. Eine inhaltliche Beschränkung auf bestimmte Erscheinungsformen von Kriminalität lässt sich diesen Vorschriften nicht entnehmen ${ }^{27}$ und so wurden in der Vergangenheit zahlreiche Rahmenbeschlüsse zur Harmonisierung des mitgliedstaatlichen Strafrechts erlassen.

Beispiele: Rahmenbeschluss zum Schutz des Euro gegen Geldfälschung $^{28}$, Rahmenbeschluss zum strafrechtlichen Schutz der Umwelt ${ }^{29}$, Rahmenbeschluss gegen Computerkriminalität ${ }^{30}$.

Die Nachfolgevorschrift des Art. 29 EUV findet sich in Art. 83 I AEUV:

(1) Das Europäische Parlament und der Rat können gemäß dem ordentlichen Gesetzgebungsverfahren durch Richtlinien Mindestvorschriften zur Festlegung von Straftaten und Strafen in Bereichen besonders schwerer Kriminalität festlegen, die aufgrund der Art oder der Auswirkungen der Straftaten oder aufgrund einer besonderen Notwendigkeit, sie auf einer gemeinsamen Grundlage zu bekämpfen, eine grenzüberschreitende Dimension haben.

Derartige Kriminalitätsbereiche sind: Terrorismus, Menschenhandel und sexuelle Ausbeutung von Frauen und Kindern, illegaler Drogenhandel, illegaler Waffenhandel, Geldwäsche, Korruption, Fälschung von Zahlungsmitteln, Computerkriminalität und organisierte Kriminalität.

Je nach Entwicklung der Kriminalität kann der Rat einen Beschluss erlassen, in dem andere Kriminalitätsbereiche bestimmt werden, die die Kriterien dieses Absatzes erfüllen. Er beschließt einstimmig nach $\mathrm{Zu}-$ stimmung des Europäischen Parlaments.

Art. 83 I UA 1 AEUV macht zur Grundvoraussetzung für eine Harmonisierung, dass die in Blick genommenen Delikte (1) der besonders schweren Kriminalität zuzuordnen sind, und dass sie (2) eine "grenzüberschreitende Dimension" haben. Sodann zählt Art. 83 I UA 2 auf, welche Kriminalitätsbereiche diese Anforderungen erfüllen. Dieser Katalog weicht teils von Art. 29 II EUV ab - so war darin der Betrug noch explizit erfasst, in Art. 83 I UA 2 AEUV findet er sich dagegen nicht mehr. Der Hauptunterschied zu Art. 29 II EUV besteht jedoch darin, dass die Liste in Art. 83 I UA 2 AEUV - vorbehaltlich einer Erweiterung gem. UA 3 - abschließend ist und so einen gewissen Ausgleich für die

23 Eingehend Weigend ZStW 116 (2004), 275, 288; Fromm StraFo 2008 , 358; Rosenau ZIS 2008, 1, 16; SAtzger IntStR, $\$ 7$ Rdn. 42 T. Walter ZStW 117 (2005), 912, 918 f. sieht auch in Art. III-267 EUV (Art. 79 AEUV) eine solche Kompetenz.

24 Für einen Vorschlag hierfür s. Tiedemann (Hrsg.) Wirtschaftsstrafrecht in der Europäischen Union, 2002.

$25 \mathrm{Zu}$ großzügig Tiedemann Wirtschaftsstrafrecht BT, Rdn. $45 \mathrm{ff}$

26 So im Ergebnis auch TiedemanN Wirtschaftsstrafrecht BT, Rdn. 48.

27 Dass Art. 31 I lit. e EUV weiter zu verstehen ist, zeigt sowohl die einleitende Formulierung des Art. 31 I (»schließt ein «) als auch Art. 29 II EUV, vgl. Streinz/Satzger EGV/EUV, Art. 31 EUV Rdn. 12; kritisch Calliess/ Ruffert/SunR Das Verfassungsrecht der Europäischen Union, 3. Aufl. 2007, Art. 31 EUV Rdn. 23.

28 ABl 2001 Nr. L 329, 2.

29 ABl 2003 Nr. L 29, 5 (vom EuGH für nichtig erklärt, Slg 2005, I-7879 - Rs C-176/03)

30 ABl 2005 Nr. L 69, 67. 
Beseitigung des Einstimmigkeitsprinzips bei der Strafrechtsharmonisierung bietet ${ }^{31}$. Die Norm wirft zwei große Fragen auf:

Zunächst deutet die Formulierung »Derartige Kriminalitätsbereiche sind « in UA 2 darauf hin, dass bzgl. der Katalogdelikte das Vorliegen der Voraussetzungen des UA 1 (besondere Schwere, grenzüberschreitende Dimension) unwiderleglich vermutet wird $^{32}$. Dann wären die Kriterien des UA 1 nur bei einer Erweiterung des Katalogs gem. Art. 83 I UA 3 (dh bei einer Modifikation des Primärrechts) und nicht bei der Ausgestaltung einer einzelnen Richtlinie (also auf Ebene des Sekundärrechts) zu berücksichtigen. Gegen diese Interpretation spricht die Systematik des Art. 83 I: Wäre in den aufgelisteten Kriminalitätsfeldern eine Rechtsangleichung unabhängig von den abstrakten Anforderungen des UA 1 möglich, dann hätte es keinen Sinn, diese in Art. 83 I an die erste Stelle zu rücken. Vielmehr hätte es genügt, sie in UA 3 als Voraussetzungen einer Erweiterung des Katalogs zu nennen. Außerdem sichern die Kriterien des Art. 83 I UA 1 die Respektierung des Subsidiaritätsgrundsatzes ab, indem sie diesem im sensiblen Bereich der Strafrechtsharmonisierung einen klareren Gehalt verleihen ${ }^{33}$. All dies spricht dafür, dass auch bei dem Erlass einer Richtlinie im Bereich des Katalogs gem. Art. 83 I UA 2 die Voraussetzungen des UA 1 zu prüfen $\operatorname{sind}^{34}$. Ganz eindeutig ist diese Lösung aber im Hinblick auf den Wortlaut nicht.

Die zweite Frage betrifft die Umstände, aus denen sich die besondere Schwere bzw. die grenzüberschreitende Dimension eines Kriminalitätsbereichs ergeben soll ${ }^{35}$. Namentlich stuft der Vertrag in Bezug auf letztere neben Art und Auswirkungen der Kriminalität die "Notwendigkeit, sie auf einer gemeinsamen Grundlage zu bekämpfen", als eigene Fallgruppe ein. Woraus diese Notwendigkeit sich aber ergeben kann, bleibt unklar, so dass die Klausel das die Unionskompetenz beschränkende Kriterium des grenzüberschreitenden Charakters zu entwerten droht. Gerade bzgl. dieser Punkte lassen sich der jüngsten Entscheidung des BVerfG interessante Anhaltspunkte entnehmen (unten IV.1.a)).

\section{bb) Strafrechtliche Annexkompetenz}

Neben der vertraglich normierten Harmonisierungskompetenz iRd 3. Säule hat der EuGH in zwei Entscheidungen neueren Datums eine strafrechtliche Annexkompetenz entwickelt, um die anderen Politikfelder der EG zu ergänzen ${ }^{36}$. Dies wurde mit Recht kritisiert, da eine vertragliche Grundlage hierfür fehlt und da so strafrechtliche Vorgaben durch Mehrheitsentscheidungen zustande kommen und Brüche in den Strafrechtssystemen überstimmter Mitgliedstaaten verursachen können ${ }^{37}$. So drohen kriminalpolitische und strafrechtsdogmatische Konzepte - Bsp: Restriktives oder liberales Strafrecht? Schuldprinzip oder »harm principle «? -, welche oftmals tief in den mitgliedstaatlichen Traditionen verwurzelt sind, ausgehebelt zu werden. Zudem könnte Strafrecht als reiner Durchsetzungsmechanismus von EG-Recht zum Einsatz kommen, was seiner ultima-ratio-Funktion zuwider liefe ${ }^{38}$. Mittlerweile sind allerdings die ersten strafrechtlichen Richtlinien im Bereich des Umweltschutzes ${ }^{39}$ und der illegalen Beschäftigung ${ }^{40}$ in Kraft getreten, so dass die Annexkompetenz der EG als anerkannt gelten muss. Während hierfür in den bisherigen Verträgen keine hinreichende Grundlage besteht, soll Art. 83 II AEUV eine explizite Regelung schaffen:

\section{(1) $(\ldots)$}

(2) Erweist sich die Angleichung der strafrechtlichen Rechtsvorschriften der Mitgliedstaaten als unerlässlich für die wirksame Durchführung der Politik der Union auf einem Gebiet, auf dem Harmonisierungsmaßnahmen erfolgt sind, so können durch Richtlinien Mindestvorschriften für die Festlegung von Straftaten und Strafen auf dem betreffenden Gebiet festgelegt werden. Diese Richtlinien werden unbeschadet des Artikels 76 gemäß dem gleichen ordentlichen oder besonderen Gesetzgebungsverfahren wie die betreffenden Harmonisierungsmaßnahmen erlassen.

Vorausgesetzt wird also zunächst, dass in dem jeweiligen Politikbereich schon anderweitige Harmonisierungsmaßnahmen erfolgt sind. Da schon bislang eine quasi unüberschaubare Zahl solcher Rechtsakte ergangen ist, bedeutet dies keine echte Einschränkung; Art. 83 II bleibt ein immenses Einfallstor für strafrechtliche EU-Vorgaben.

Beispiele ${ }^{41}$ : Strafrechtliche Rahmenvorschriften kommen als Annex $\mathrm{zu}$ anderweitigen Kompetenzen der EU zukünftig auch in Betracht im Bereich des Verkehrsrechts (Art.91 I AEUV), des Wettbewerbsrechts (Art. 103 I AEUV), des Gesundheitswesens (Art. 168 IV AEUV), des Energierechts (Art. 194 II AEUV) oder auch umfassend zur Verwirklichung des Gemeinsamen Binnenmarktes (Art. 114 I AEUV).

Eingrenzen lässt sich die Annexkompetenz allenfalls über das Kriterium der Unerlässlichkeit, welches sich als besonders strenge Ausprägung des Subsidiaritätsgrundsatzes begreifen lässt ein Weg, den auch das BVerfG einschlägt (unten IV.1.b)). Teils wird angeregt, die Voraussetzungen des Art. 83 I AEUV bzgl. der zu bekämpfenden Straftaten (besondere Schwere, grenzüberschreitender Charakter) hierauf zu übertragen ${ }^{42}$. Wie bereits angedeutet, sind diese jedoch ihrerseits problematisch. Auch zeigt die (vorausgesetzte) Existenz anderweitiger Harmonisierungsmaßnahmen, dass die Hürde der Subsidiarität auf dem betreffenden Gebiet schon einmal überwunden werden konnte. Bei der Auslegung der »Unerlässlichkeit« sollte deshalb in jedem Fall auch der spezifische Bedarf gerade an strafrechtlichen Sanktionen eine tragende Rolle spielen.

\section{Kompensationsversuche}

Im Lissabon-Vertrag sind auch einige Instrumente vorgesehen, um die Stärkung supranationaler Elemente und den damit einhergehenden Bedeutungsverlust des einzelnen Staates (gerade auf dem Gebiet des Strafrechts) zumindest teilweise auszugleichen.

\section{a) Verstärkte Mitwirkungsrechte der nationalen Parlamente}

Zunächst wird die Rolle der nationalen Parlamente durch eine intensivere Einbeziehung in den europäischen Rechtssetzungsprozess gestärkt. Gem. Art. 2 des neuen Protokolls Nr. $1^{43}$ sind sie über europäische Rechtsaktsvorhaben praktisch zeitgleich mit den EU-Organen zu informieren; um ihnen Gelegenheit zur Stellungnahme zu geben, darf über eine solche Initiative idR auch erst nach acht Wochen abgestimmt werden (vgl. Art. 4 des Protokolls). Noch klarer umrissen sind die Mitspracherechte in dem neuen Protokoll Nr. $2^{44}$ über die Grundsätze der Subsidiarität $t^{45}$.

31 Vedder/Heintschel von Heinegg/Kretschmer Europäischer Verfassungsvertrag, 2007, Art. III-271 Rdn. 6.

32 So etwa die Befürchtung von Weigend ZStW 116 (2004), 275, 283.

33 Vedder/Heintschel von Heinegg/Kretschmer EVV, 2007, Art. III-271 Rdn. 4.

34 T. WAlter ZStW 117 (2005), 912, 926.

35 Kritisch zur Unklarheit dieses Begriffs auch WeIgend ZStW 116 (2004), 275, 283.

36 EuGH Slg 2005, I-7879 - Rs C-176/03 (Umweltstrafrecht) sowie Slg 2007, I-9097 - Rs. C-440/05 (Meeresverschmutzung).

37 Hefendehl ZIS 2006, 161; Pohl ZIS 2006, 213; Heger JZ 2006, 310; ZimmermanN NStZ 2008, 662; Zöller ZIS 2009, 340; SATZger IntStR, $\$ 8$ Rdn. 41 ff.; Амв оs IntStR, $\$ 11$ Rdn. 30 a ff.; aufgeschlossener DANNECKER JURA 2006, 95, 98; HeCKer EUStR, $\$ 8$ Rdn. 27; Eisele JZ 2008, 251; SuHr ZEuS 2008, 45, 57 ff.

38 SATZGER in: 4. Europäischer Juristentag, 2008, 207, 216; KAIAFA-GBANDI ZIS 2006, 521, 524.

39 RL 2008/99/EG, ABl 2008 Nr. L 330, 28 (dazu Zimmermann ZRP 2009, $74 \mathrm{ff}$.).

40 RL 2009/52/EG, ABl 2009 Nr. L 168, 24 (dazu ZimmermanN ZIS 2009, 1, $8 \mathrm{ff}$.).

41 S. auch die Auflistung bei Tiedemann Wirtschaftsstrafrecht BT, Rdn. 53, die auf den Vorschlägen für »Europa-Delikte« in Tiedemann (Hrsg.), Wirtschaftsstrafrecht in der Europäischen Union, 2002, beruht.

42 So Schünemann in: ders. (Hrsg.), Alternativentwurf Europäische Strafverfolgung, 2004, 75, 81; T. WALter ZStW 117 (2005), 912, 929; BACIGALUPo ZStW 116 (2004), 326, 329.

$43 \mathrm{ABl} 2008$ Nr. C 115, 203.

44 ABl 2008 Nr. C 115, 206.

45 S. zu diesem Komplex auch Haratsch/Koenig/Pechstein Europarecht, Rdn. $62 \mathrm{~d}$ ff. 
Zum einen kann gem. Art. 7 II ein Viertel der nationalen Parlamente das Organ, von dem ein Vorschlag mit Strafrechtsbezug ausgeht, dazu zwingen, eine begründete Stellungnahme zur Wahrung des Subsidiaritätsgrundsatzes abzugeben. Äußert gar eine Mehrheit der nationalen Parlamente Zweifel im Hinblick auf den Subsidiaritätsgrundsatz, müssen nach Art. 7 III auch der Rat sowie das Europäische Parlament dies bei ihrer Beschlussfassung berücksichtigen. Letztlich hat die Stellungnahme der nationalen Parlamente aber nicht viel mehr als eine Appellfunktion. Außerdem sollte es innerhalb einer Rechtsordnung, die so maßgeblich auf dem Subsidiaritätsprinzip beruht wie die europäische, eine Selbstverständlichkeit sein, dass die Legislative diesem Grundsatz auch ohne gesonderte Aufforderung von dritter Seite (durch die nationalen Parlamente) Rechnung trägt. Immerhin wird künftig aber der EuGH für Nichtigkeitsklagen wegen der Verletzung des Subsidiaritätsgrundsatzes durch strafrechtliche Rechtsakte zuständig sein ${ }^{46}$ - insoweit ist die Wahrung der Subsidiarität bislang nicht justitiabel ${ }^{47}$.

\section{b) "Notbremse" gegen Harmonisierungsmaßnahmen}

Mit der sog. "Notbremse« findet sich ein besonderer Mechanismus im jeweiligen Abs. 3 von Art. 82 und Art. 83 AEUV, die wie gesehen eine Harmonisierung des mitgliedstaatlichen Straf- und Strafprozessrechts ermöglichen. Ein Staat, der durch eine solche Richtlinie "grundlegende Aspekte seiner Strafrechtsordnung" berührt sieht, kann das ordentliche Gesetzgebungsverfahren blockieren und sich einer Rechtsangleichung entziehen. Die übrigen Mitgliedstaaten können jedoch den betreffenden Rechtsakt im Wege einer verstärkten Zusammenarbeit erlassen, so dass dieser nur ihnen gegenüber Wirkung entfaltet.

Von maßgeblicher Bedeutung wird dabei sein, welche Anforderungen an die "grundlegenden Aspekte" gestellt werden. Als Ausgangspunkt kann dafür dienen, dass nur jeder Mitgliedstaat selbst beurteilen kann, welche Inhalte seines Strafrechts so zentral sind, dass er sie nicht preisgeben darf. Zu erwarten ist aber, dass der EuGH das Vertragsverletzungsverfahren gem. Art. $258 \mathrm{ff}$. AEUV zumindest für eine Missbrauchskontrolle nutzen und prüfen wird, ob sich ein Mitgliedstaat ohne hinreichende Grundlage auf Art. 82 III, 83 III AEUV beruft und dadurch seine Treuepflicht aus Art. 4 III EUV nF (Art. 10 EGV) verletzt. Fraglich erscheint darum insbesondere, inwiefern auch die Vereinheitlichung einzelner Tatbestände durch das Ziehen der "Notbremse» vereitelt werden kann: So lässt sich in jeder Ausdehnung von Strafvorschriften ein Verstoß gegen den ultima-ratio-Grundsatz erblicken; auch eine Verletzung des Bestimmtheitsgebots liegt häufig nicht fern. In diesen Fällen kann nur ein Vergleich mit Tatbeständen des jeweiligen Mitgliedstaats weiterhelfen, die eine ähnliche Schutzrichtung aufweisen: Sind zB Vorbereitungshandlungen oder der Versuch bei vergleichbaren Delikten ebenfalls unter Strafe gestellt? Oder: Wie ist das Strafmaß bei ähnlichen Tatbeständen ausgestaltet? Leichter dürfte die Argumentation fallen, wenn europäische Vorgaben direkt den Allgemeinen Teil oder strafrechtliche Grundprinzipien betreffen ${ }^{48}$. In jedem Fall muss aber den staatlichen Stellen ein erheblicher Einschätzungsspielraum belassen werden.

\section{Allgemeine Aussagen des BVerfG-Urteils}

In dem Verfahren vor dem BVerfG rügten die Beschwerdeführer einen Verlust der Staatlichkeit der Bundesrepublik und ein Defizit in der demokratischen Legitimation der Union. Sie stützten diesen Vorwurf auf ihr Wahlrecht gem. Art. 38 I GG: Dieses grundrechtsgleiche Recht würde seines Inhalts beraubt, da nach dem Vertrag von Lissabon die entscheidenden Weichenstellungen der Politik in Brüssel getroffen würden, ohne dass dies durch eine adäquate demokratische Legitimation auf europäischer Ebene kompensiert sei. Die Karlsruher Richter teilen diese Deu- tung zumindest im Ergebnis nicht. Kurz gesagt verfolgen sie dabei drei Argumentationsstränge.

\section{Keine "staatsanaloge« Ausgestaltung der Union}

Erstens stuft das BVerfG die EU auch weiterhin ${ }^{49}$ als eine Form der zwischenstaatlichen Zusammenarbeit (als »Staatenverbund «) und gerade nicht als Bundesstaat ein: Weder komme im Lissabon-Vertrag ein auf Staatsgründung gerichteter Wille zum Ausdruck, noch würden die demokratischen Strukturen den Anforderungen gerecht, welche an die Bundesebene in einem Bundesstaat zu stellen seien (Rdn. 277). So komme es nicht zu einem echten Wettstreit zwischen Regierung und Opposition, wie er für parlamentarische Demokratien typisch sei (Rdn. 280). Insbesondere aber fehle es bei der Wahl zum Europäischen Parlament an der Erfolgschancengleichheit aller Stimmen, weil kleine Mitgliedstaaten (zB Luxemburg, Malta) im Verhältnis zu ihrer Bevölkerungszahl mehr Abgeordnete entsenden als größere Mitgliedstaaten (Rdn. 284 f.). Für ihre nicht »staatsanaloge« Organisationsform ${ }^{50}$ sei die demokratische Legitimation der EU jedoch ausreichend (Rdn. 278).

\section{Kein untragbarer Souveränitätsverlust Deutschlands}

Zweitens betont das BVerfG, dass unter Geltung des Grundgesetzes wesentliche Elemente souveräner Staatlichkeit bei der Bundesrepublik verbleiben müssten (Rdn. $226 \mathrm{ff}$.). Insbesondere sei ein Beitritt Deutschlands zu einem europäischen Bundesstaat nur mit dem unmittelbar erklärten Willen des deutschen Volkes - also mittels eines Referendums über eine neue Verfassung möglich (Rdn. 228; s. auch Art. 146 GG). Allerdings sehen die Richter in dem neuen Vertragswerk keinen Verstoß gegen diese Grundsätze (Rdn. 298 ff.). Die generelle Abgrenzung der Zuständigkeiten von Mitgliedstaaten und Union folge nämlich weiterhin dem Prinzip der begrenzten Einzelermächtigung (Rdn. 301). Zudem garantierten die Grundsätze der Subsidiarität und der Verhältnismäßigkeit (»Kompetenzausübungsschranken«) weiterhin, dass wesentliche Elemente der deutschen Staatsgewalt erhalten blieben (Rdn. 304 f.).

Kritik formuliert das Gericht bzgl. Vorschriften, die unter vereinfachten Bedingungen Vertragsänderungen ermöglichen sollen (zB die sog Brückenklauseln): Deutsche Verfassungsorgane müssten bei der Übertragung von Kompetenzen sicherstellen, dass sowohl das politische System der Bundesrepublik als auch das der EU weiterhin elementaren demokratischen Anforderungen entsprechen und so ihrer "Integrationsverantwortung " gerecht werden (Rdn. 245). Eine Vertragsänderung ohne nationales Ratifikationsverfahren ebnete dagegen den Weg für eine Übertragung von Zuständigkeiten ohne explizite Genehmigung des Bundestags. Das Zustimmungsgesetz zum Lissabon-Vertrag selbst - mit dem ja auch die Klauseln zur Vertragsänderung allgemein gebilligt werden - genüge hierfür nicht, weil nicht hinreichend vorhersehbar sei, wie weit diese reichen könnten (Rdn. 317 ff., 326 ff.). Deshalb müsse das deutsche Begleitgesetz gewährleisten, dass ein solcher Schritt nicht ohne das Parlament möglich sei (Rdn. 239, $409 \mathrm{ff}$.). Da es diesen Anforderungen nicht gerecht werde, sei es verfassungswidrig, so dass Deutschland erst nach entsprechenden Nachbesserungen (unten IV.3.) den Lissabon-Vertrag ratifizieren dürfe.

Um einem verfassungswidrigen Souveränitätsverlust vorzu-

46 Skeptisch bzgl. der Überprüfbarkeit des Subsidiaritätsprinzips zB HerDEGEN Europarecht, $\$ 7$ Rdn. 27.

47 Art. 46 EUV erstreckt die Zuständigkeit des EuGH nicht auf den in Art. 2 II EUV niedergelegten Subsidiaritätsgrundsatz.

48 Dannecker JURA 2006, 173, 177; s. auch unten IV.1.c).

49 So bereits eine der zentralen Aussagen des Maastricht-Urteils, BVerfGE 89, 155, Ls. 8.

50 S. hierzu auch Herdegen Europarecht, $\$ 6$ Rdn. $16 \mathrm{f}$. 
beugen, behält es sich das BVerfG - wie schon im KloppenburgBeschluss $^{51}$ - vor, EU-Rechtsakte trotz deren prinzipiellen Vorrangs auf ihre Verfassungsmäßigkeit zu überprüfen (Rdn. 240 ff., $331 \mathrm{ff}$.). Zum einen erlaube dies eine »Identitätskontrolle« daraufhin, ob EU-Recht Kerninhalte des Grundgesetzes in Frage stelle. Zum anderen soll davon auch eine "ultra-vires-Kontrolle« umfasst sein, um sicherzustellen, dass die EU nicht jenseits ihrer Befugnisse handle. Auf diesem Weg sollen besonders die Einhaltung des Prinzips der begrenzten Einzelermächtigung und des Subsidiaritätsprinzips gewährleistet werden. Dies begründen die Richter mit der These, selbst höherrangiges Recht sei zur Selbstbehauptung der Identität einer Rechtsgemeinschaft überprüfbar. Dabei berufen sie sich interessanterweise auch auf das viel beachtete Kadi-Urteil des EuGH ${ }^{52}$ (Rdn. 340). Darin hatte der Gerichtshof eine EG-Verordnung ${ }^{53}$ wegen eines Verstoßes gegen Grundrechte ${ }^{54}$ für nichtig erklärt, obwohl sie auf Resolutionen des UN-Sicherheitsrats ${ }^{55}$ zurückging. Zumindest dieser Vergleich lässt sich aber anzweifeln: Die EG ist nicht selbst Mitglied der UNO und ein unmittelbarer Vorrang von UN-Resolutionen (nicht von allgemeinen Grundsätzen des Völkerrechts ${ }^{56}$ ) gegenüber im Primärrecht verankerten Grundrechten lässt sich deshalb schwer konstruieren.

\section{Zuständigkeit zur Regelung zentraler Lebensbereiche}

Drittens leitet das BVerfG aus dem Demokratieprinzip - neben der Forderung nach einem Erhalt der nationalen Souveränität zusätzliche inhaltliche Grenzen für die Übertragung von Hoheitsrechten ab (Rdn. 249). Dass mit dem Lissabon-Vertrag erneut Kompetenzen an die EU abgegeben werden, sei zwar nicht per se problematisch, da es kein festlegbares Minimum an Zuständigkeiten gebe, welches den Nationalstaaten verbleiben müsse (Rdn. 248). Das Gericht wiederholt jedoch die Aussage des Maastricht-Urteils, das deutsche Parlament müsse auch nach dem Inkrafttreten der Vertragsreform die Letztentscheidungsgewalt in zentralen Lebensbereichen behalten ${ }^{57}$. An dieser Stelle wird das Urteil für das (Europäische) Strafrecht besonders interessant: Neben den Außenwirtschaftsbeziehungen, der Verteidigungs- und der Sozialpolitik zählt das BVerfG zu diesem Kern nationaler Entscheidungsbefugnisse insbesondere auch die Strafrechtspflege.

\section{Die Bedeutung des BVerfG-Urteils speziell für das Europäische Strafrecht}

Das BVerfG billigt zwar prinzipiell die Übertragung zusätzlicher Strafrechtskompetenzen auf die EU, jedoch nur unter Auflagen hinsichtlich der Auslegung der neuen Vorschriften. Hinsichtlich der demokratischen Legitimation ${ }^{58}$ liegt eine These allen weiteren Passagen des Urteils mit Strafrechtsbezug zugrunde: Das BVerfG zählt das Strafrecht wegen der damit verbundenen massiven Grundrechtseingriffe und seiner stark kulturellen und historischen Prägung zum Kern der nationalen Identität, über den das Volk in einer Demokratie grundsätzlich selbst bestimmen können müsse (Rdn. 249, 355 f.). Damit greift es den ursprünglich von Satzger aufgestellten »strafrechtsspezifischen Schonungsgrundsatz « auf, demzufolge jede Harmonisierung des Strafrechts mit besonderer Rücksicht auf die nationalen Besonderheiten vonstatten gehen muss ${ }^{59}$.

\section{Anforderungen an die Auslegung einzelner Kompetenz- normen}

Aus der Forderung, dass die elementaren Lebensbereiche nicht überwiegend auf EU-Ebene normiert werden dürften (Rdn. 264), leitet das BVerfG ein Gebot der restriktiven Auslegung strafrechtlicher Harmonisierungs-Kompetenzen ab, deren Nutzung stets einer besonderen Rechtfertigung bedürfe (Rdn.358). Hierbei bleibt das Gericht jedoch nicht stehen; vielmehr macht es teils konkrete Vorgaben für die Auslegung einzelner Vorschriften. Die- se können natürlich weder den EU-Gesetzgeber noch den EuGH als Organe der eigenständigen EU-Rechtsordnung (die grundsätzlich einen höheren Rang als diejenige der Mitgliedstaaten genießt ${ }^{60}$ rechtlich binden. Faktisches Gewicht erhalten sie aber im Zusammenhang mit der Reserve-Überprüfungszuständigkeit, welche das BVerfG sich im Hinblick auf EU-Rechtsakte vorbehält. Werden Rechtsakte mit Strafrechtsbezug in weiterem Umfang erlassen, als das BVerfG es in seinem Urteil gebilligt hat, könnte das Gericht tatsächlich zu der von ihm angedrohten Identitäts- bzw. ultra-vires-Kontrolle schreiten.

\section{a) Besonders schwere Kriminalität, Art.83 I AEUV}

aa) Voraussetzungen einer Harmonisierung

(1) Wie gesehen geht aus dem Vertragstext nicht eindeutig hervor, ob die Voraussetzungen der besonderen Schwere und der grenzüberschreitenden Dimension bei dem Erlass einer Richtlinie iRd Katalogs in Art. 83 I UA 2 AEUV noch gesondert zu prüfen sind. Das BVerfG setzt sich mit der Frage nicht explizit auseinander. Einige - wenngleich etwas ungeordnete - Anhaltspunkte finden sich jedoch in Rdn. 363: Die Richter stellen als Anknüpfungspunkte für eine restriktive Auslegung des Art. 83 I die Zusammensetzung des Katalogs in UA 2 und die Kriterien des UA 1 nebeneinander. Außerdem bezeichnen sie die aufgelisteten Delikte als »typischerweise - also nicht ausnahmslos - grenzüberschreitend. Die Bemerkung, europäische Rahmenvorschriften dürften nur die grenzüberschreitende Dimension eines konkreten Straftatbestands ansprechen (Rdn. $363 \mathrm{aE}$ ), deutet ebenfalls in diese Richtung. Zwar könnte sie sich (wegen eines vorangehenden Einschubs) allein auf die Erweiterungsklausel gem. Art. 83 I UA 3 AEUV beziehen. Jedoch nimmt das BVerfG auf die Ausgestaltung konkreter Tatbestände Bezug, während Art. 83 I UA 3 eine Erweiterung des UA 2 um ganze Kriminalitätsbereiche erlaubt. All dies legt den Schluss nahe, dass das BVerfG entsprechend der hier vertretenen Auffassung - die Kriterien des Art. 83 I UA 1 auch bei dem Erlass einer einzelnen Richtlinie im Bereich der Katalogdelikte überprüft sehen will.

(2) Deutlicher wird das BVerfG im Hinblick auf die zweite wichtige Frage iRd Art. 83 I AEUV - die Auslegung der »besonderen Notwendigkeit « einer gemeinsamen Bekämpfung von Straftaten. Diese soll sich demnach ausschließlich aus Art oder Auswirkungen der betreffenden Straftaten ergeben können (Rdn. 359). Damit »korrigiert « das BVerfG aber den Vertragstext, welcher die Notwendigkeit gemeinsamen Handelns mit Art und Auswirkungen des betreffenden Delikts auf eine Stufe stellt. Mag dies auch noch so sinnvoll sein, die Grenzen der Auslegung werden damit überschritten, und noch dazu auf der Ebene des prinzipiell Vorrang beanspruchenden EU-Primärrechts. Überzeugender sind die Negativbeispiele für Erwägungen, die keinesfalls eine beson-

51 BVerfGE 75, 223, 235, 242

52 EuGH, verb. Rs C-402/05 P und C-415/05 P, Urt. v. 3. 9. 2008.

53 VO (EG) 881/2002, ABl 2002 Nr. L 139, 9. Diese betraf das Einfrieren von Geldern, welche Osama bin Laden oder dem Terrornetzwerk Al Qaida zugute kommen können.

54 Einerseits das Grundrecht auf rechtliches Gehör (Rdn. 333 ff. des KadiUrteils), andererseits das Grundrecht auf Eigentum (Rdn. 354 ff. des Kadi-Urteils).

55 Res. 1267 (1999) v. 15. 10. 1999 und Res. 1333 (2000) v. 19.12. 2000.

56 Die Bindung hieran kann aus der Eigenschaft der EU als Völkerrechtssubjekt abgeleitet werden, hierzu (schon de lege lata) Oppermann/ Classen/Nettesheim Europarecht, 4. Aufl. 2009, \$6 Rdn. 7.

57 BVerfGE 89, 155, 207.

58 S. hierzu auch SIEBER ZStW 121 (2009), 1, 58: Legitimation der Harmonisierungskompetenzen ausreichend.

59 SAtZger Die Europäisierung des Strafrechts, 2001, 166 ff.; DERs. IntStR, $\$ 8$ Rdn. 9; ebenso Hecker EUStR $\$ 8$ Rdn. 70; Eisele JZ 2001, 1160, 1163.

60 EuGH, Slg 1964, 1251, Rs 6/64 (Costa ./. ENEL), Rdn. 8 ff. 
dere Notwendigkeit iSd Art. 83 I UA 1 AEUV begründen dürfen: So könne allein der politische Wille der Vertragsstaaten hierfür nicht ausreichen (Rdn. 359). Zudem erteilt das BVerfG der Praxis eine Absage, den Bedarf an strafrechtlicher Harmonisierung in europäischen Rechtsakten damit zu begründen, dieser Schritt diene der Vereinfachung der internationalen Zusammenarbeit bei der Strafverfolgung ${ }^{61}$. Die Erleichterung des Rechtshilfeverkehrs kann nämlich kein Selbstzweck sein, welcher eine Verschärfung von Strafvorschriften legitimieren würde ${ }^{62}$ :

"Das Strafrecht in seinem Kernbestand dient nicht als rechtstechnisches Instrument zur Effektuierung einer internationalen Zusammenarbeit, sondern steht für die besonders sensible demokratische Entscheidung über das rechtsethische Minimum.« (Rdn. 358)

\section{bb) Harmonisierungsdichte}

Das Urteil des BVerfG ermöglicht nicht nur Schlussfolgerungen im Hinblick auf die Voraussetzungen (das »Ob«) einer Harmonisierung, sondern auch bzgl. der zulässigen Intensität (das »Wie«) strafrechtlicher EU-Vorgaben, also der »Harmonisierungsdichte $\ll$.

(1) In einem Nebensatz fordert das BVerfG »substantielle Ausgestaltungsspielräume « für die Mitgliedstaaten, und zwar umso mehr, je enger strafrechtliche Rahmenvorschriften stark historisch oder traditionell geprägte Bereiche betreffen (Rdn. 363). Eine klare Grenze für die noch akzeptable Harmonisierungsdichte stellt dies natürlich nicht dar. Zumindest aber darf die EU den Mitgliedstaaten danach keine komplett ausformulierten Strafvorschriften vorgeben. An sich wäre das auf Grund der Zielverbindlichkeit von Richtlinien bzw. Rahmenbeschlüssen eine Selbstverständlichkeit; gleichwohl zeigen sich heute bereits Ansätze zu einer solchen strafrechtlichen $»$ Totalharmonisierung ${ }{ }^{63}$. Auch wenn der EU-Gesetzgeber den Mitgliedstaaten einen $\mathrm{Ge}$ staltungsspielraum nur auf Straffolgenseite beließe, also zB einen Tatbestand vollständig vorgäbe, Art und Maß der Strafe aber offen ließe, würde dies wohl nicht genügen: Das BVerfG stellt auf die wertegebundene Entscheidung über die Strafbarkeit von Verhaltensweisen und damit eher auf die Tatbestandsseite ab. Sobald eine Richtlinie zwingend eine kriminalstrafrechtliche Sanktion vorschreibt (egal, ob sie Vorgaben bzgl. deren Art oder Höhe enthält) und nicht auch administrative oder zivilrechtliche Sanktionen genügen lässt, muss sie den Mitgliedstaaten deshalb auf der Tatbestandsseite Gestaltungsmöglichkeiten belassen. Wann diese noch »substantiell « sind, muss sich aber aus den Umständen des Einzelfalles ergeben. Dabei lässt sich insbesondere auf die Voraussetzungen zurückgreifen, welche gem. Art. 83 I UA 1 AEUV die Unionskompetenz begründen: Je gravierender die Folgen eines Kriminalitätsbereichs sind und je stärker sich ein grenzüberschreitender Bezug - etwa auf Grund der Organisationsstruktur der Täter - feststellen lässt, desto detaillierter dürfen auch die strafrechtlichen EU-Rahmenvorschriften sein.

(2) Schließlich gibt das BVerfG noch einen »Tipp«, wie sich die mitgliedstaatliche Strafrechtskompetenz schonen lasse: Europäische Mindestvorschriften sollten nicht einen kompletten Deliktsbereich, sondern nur einzelne Tatbestandsvarianten erfassen, die den von Art. 83 I UA 1 geforderten grenzüberschreitenden Charakter aufweisen (Rdn. $363 \mathrm{aE})^{64}$. Als Beispiel dafür, wie der EU-Gesetzgeber nicht vorgehen sollte, nennt es dabei Art. 2 II des Rahmenbeschlusses über den Europäischen Haftbefehl ${ }^{65}$. Ein geeignetes Beispiel ist das kaum, da dieser Rechtsakt nicht auf die Harmonisierung des materiellen Strafrechts, sondern auf die Reformierung des traditionellen Auslieferungsverfahrens gerichtet war. Abgesehen davon ist nicht recht ersichtlich, wie die Äußerung gemeint ist, da jeder Rahmenbeschluss zur Angleichung des materiellen Rechts einzelne Verhaltensweisen (und in diesem Sinne »Tatbestandsvarianten«) benennt, für deren Bestrafung die Mitgliedstaaten zu sorgen haben. Zumindest aber macht die Passage noch einmal deutlich, dass die eu- ropäischen Akteure bei der Harmonisierung des Strafrechts besondere Rücksicht walten lassen müssen.

\section{b) Annexkompetenz, Art. 83 II AEUV}

Die EuGH-Rechtsprechung zur strafrechtlichen EG-Annexkompetenz hatte Anlass zu der Sorge gegeben, dass Strafrecht künftig nur noch als reiner Durchsetzungsmechanismus für EG-Politiken dienen und eine massive Harmonisierungstätigkeit auf dem Gebiet des Strafrechts ermöglichen könnte (oben II.3.b)bb)). Insoweit findet das BVerfG sehr klare Worte:

»Hinter der Annexzuständigkeit verbirgt sich eine gravierende Ausdehnung der Zuständigkeit zur Strafrechtspflege im Vergleich zur bislang geltenden Rechtslage.... Wegen drohender Uferlosigkeit dieses die Strafrechtssetzung betreffenden Kompetenztitels ist eine solche Kompetenzvorschrift mit dem Prinzip einer sachlich bestimmten und nur begrenzten Übertragung von Hoheitsrechten an sich ebenso wenig zu vereinbaren wie mit dem gebotenen Schutz des demokratisch an die Mehrheitsentscheidung des Volkes besonders rückgebundenen nationalen Gesetzgebers.«(Rdn. 361)

\section{aa) Das Kriterium der Unerlässlichkeit}

Dass sich die Vorschrift überhaupt mit dem Grundgesetz vereinbaren ließe, sei nur auf die Möglichkeit einer restriktiven Auslegung anhand des Merkmals der Unerlässlichkeit zurückzuführen. So fordert das BVerfG: "Damit dieser Ausnahmetatbestand erfüllt ist ..., muss nachweisbar feststehen, dass ein gravierendes Vollzugsdefizit tatsächlich besteht und nur durch Strafdrohung beseitigt werden kann. ${ }^{66} \mathrm{Ob}$ diese - sicher zustimmungswürdige - Stellungnahme viel bewirken wird, ist angesichts der bisherigen EuGH-Rechtsprechung allerdings fraglich: Darin wurde die Unerlässlichkeit strafrechtlicher Maßnahmen ebenfalls vorausgesetzt, aber nicht ernsthaft geprüft ${ }^{67}$. Einen Seitenhieb hierauf kann sich das BVerfG denn auch nicht verkneifen und es stellt trocken fest, diese Anforderungen müssten auch iRd durch den EuGH begründeten EG-Annexkompetenz erfüllt sein. Gleichwohl lässt sich festhalten, dass es angesichts der Schwierigkeiten, das Kriterium der Unerlässlichkeit strafrechtlicher Sanktionen zu konkretisieren, schwer fällt, eine sinnvolle Einschränkung der Annexkompetenz zu erzielen. Im Zweifel wird man sich deshalb auf die »Notbremse « in Art. 83 III AEUV und die ultra-vires-Kontrolle des BVerfG verlassen müssen.

\section{bb) Die zulässige Harmonisierungsdichte}

Unklar ist, ob das BVerfG daneben auch Vorgaben in Bezug auf die zulässige Harmonisierungsintensität (das »Wie«) machen wollte. Zumindest ausdrücklich bezieht sich das Erfordernis wesentlicher Ausgestaltungsspielräume (Rdn. 363) nur auf Art. 83 I AEUV. Dies deutet darauf hin, dass das BVerfG im Bereich der Annexkompetenz eine höhere Regelungsdichte akzeptieren würde. Dies wäre insofern nachvollziehbar, als Art. 83 II AEUV voraussetzt, dass der betreffende Bereich schon anderweitig harmonisiert worden ist. Jedoch könnten wegen des Wertebezugs des Strafrechts gleichwohl erhebliche Wertungsunterschiede zwischen den Mitgliedstaaten auftreten. Der unmissverständlich geforderte Respekt vor den nationalen Strafrechtsordnungen

61 Vgl. Erwägungsgrund 4 des Rahmenbeschlusses gegen Rassismus und Fremdenfeindlichkeit, ABl 2008 Nr. L 328, 55.

62 In diesem Sinne auch KaIAfa-GBandi ZIS 2006, 521, $522 \mathrm{f}$.

63 Kaiafa-Gbandi KritV 2004, 3, 4 f.; Dannecker JURA 2006, 95, 98.

64 Auch hier ist nicht ganz klar, ob sich das BVerfG nicht nur auf die Ergänzungsklausel in UA 3 bezieht. Durch eine Ausdehnung des Katalogs in UA 2 würden aber noch keine "Mindestvorschriften " geschaffen; s. außerdem IV.1.a)aa).

65 ABl 2002 Nr. L 190, 1.

66 Rdn. 362 des Urteils; Hervorhebungen stammen vom Verfasser.

67 Dazu Zimmermann NStZ 2008, 662, 664 f; s. auch schon Schünemann in: ders. (Hrsg.), Alternativentwurf, $75 \mathrm{f}$. 
verlangt es deshalb, bei einem Tätigwerden iRd Art. 83 II AEUV die Traditionen der Mitgliedstaaten auch in Bezug auf das "Wie« so weit als möglich zu schonen ${ }^{68}$.

\section{c) Betonung des Schuldprinzips}

Schließlich zieht das BVerfG eine absolute Grenze für jegliche Strafrechtsangleichung: Europäische Vorgaben dürften Deutschland nicht zu einer Aufgabe des verfassungsrechtlich (in Art. 1 I GG) verankerten Schuldprinzips zwingen (Rdn. 364). Dies liefert insbesondere einen inhaltlichen Anhaltspunkt für den Gebrauch der "Notbremse« in Art. 83 III AEUV: Jedenfalls wenn Anweisungen in einer Richtlinie nicht mit dem Schuldprinzip deutscher Prägung zu vereinbaren sind - also zB eine strafrechtliche Sanktionierung juristischer Personen vorschreiben ${ }^{69}$-, darf sich Deutschland an dem Rechtsakt nicht beteiligen.

\section{Bedeutung des Urteils auch für supranationales Strafrecht?}

Die bisher dargestellten Aussagen des Urteils bezogen sich auf die Auslegung strafrechtlicher Harmonisierungskompetenzen. Sie lassen sich nicht direkt auf die Schaffung eines originär europäischen Betrugsstrafrechts gem. Art. 325 IV AEUV übertragen, weil dadurch die Strafrechtskompetenz der Mitgliedstaaten formal unberührt bliebe ${ }^{70}$. Höchstens indirekt würde durch europäische Tatbestände, die naheliegenderweise die nationalen Strafvorschriften verdrängen müssten ${ }^{71}$, die Regelungszuständigkeit der Mitgliedstaaten beschnitten. Nur die Passage zum Schuldprinzip (Rdn. 364) bezieht sich nicht ausdrücklich auf die Befugnisse zur Rechtsangleichung, sondern auf »Zuständigkeiten ... im Bereich der Strafrechtspflege«. Auch sie ist aber ganz in den Kontext des Art. 83 AEUV eingebettet.

Somit lassen sich für die Setzung von supranationalem Strafrecht nur die allgemeinen Maximen, auf denen das Urteil basiert, fruchtbar machen. Dies sind in erster Linie die kurz skizzierten demokratietheoretischen Erwägungen. Da Art. 325 IVAEUV die Befugnisse der EU hinreichend klar bestimmt und das deutsche Parlament insofern seine »Integrationsverantwortung « wahrnehmen konnte, sollten sie einer Strafrechtssetzung durch die EU nicht per se im Wege stehen. Wenn das Strafrecht aber besonderer demokratischer Legitimation bedarf und die EU nicht das demokratische Niveau eines Staates erreicht, dann muss sie hierbei jedenfalls zurückhaltend zu Werke gehen. Zumindest das Gebot restriktiver Auslegung lässt sich so auf Art. 325 AEUV erstrecken und stützt eine eher restriktive Interpretation dieser Norm (s. II.3.a)). Auf Grund der indirekten Beeinflussung des mitgliedstaatlichen Kompetenzraums durch die Schaffung europäischer Straftatbestände muss zudem das Subsidiaritätsprinzip peinlichst genau respektiert werden ${ }^{72}$.

\section{Die formale Seite: Mitwirkungsrechte des Bundestags}

Zudem entfalten die oben dargestellten Anforderungen an eine ausreichende demokratische Legitimation von Kompetenzübertragungen auch Wirkungen für die Parlamentsbeteiligung an einzelnen Entscheidungen mit Strafrechtsbezug auf europäischer Ebene.

\section{a) Gebrauch von Blankettermächtigungen}

Hinsichtlich der Generalklauseln in Art. 82 II lit. d und Art. 83 I UA 3 AEUV kritisiert das BVerfG, sie würden eine Erweiterung des strafrechtlichen Harmonisierungsprogramms ohne genügende demokratische Rückkopplung durch die nationalen Parlamente gestatten ${ }^{73}$. Dasselbe gelte für Art. 86 IV AEUV, demzufolge der Zuständigkeitsbereich einer künftigen Europäischen Staatsanwaltschaft ausgedehnt werden kann. Der deutsche Vertreter im Rat dürfe deshalb einer Nutzung dieser Blankettermächtigungen nur zustimmen, wenn der Bundestag dies zuvor mit einem Gesetz gem. Art. 23 I 2 GG abgesegnet hat (Rdn. 419). Auch eine Ablösung des in diesen Vorschriften vorgesehenen
Einstimmigkeitserfordernisses gem. Art. 48 VII EUV nF setze demnach ein solches Zustimmungsgesetz voraus.

b) Gebrauch der »Notbremse « in Art. 82 III, 83 III AEUV

Darüber hinaus fordert das BVerfG eine Beteiligung des Parlaments, wenn es um einen Gebrauch der strafrechtlichen »Notbremse« geht (Rdn. 418): Der deutsche Ratsvertreter dürfe bzgl. Art. 82 III und Art. 83 III AEUV »nur auf Weisung des Bundestags ... handeln«. Diese Formulierung ist missverständlich, klingt sie doch so, als müsse sich der Ratsvertreter das Ziehen der »Notbremse« gestatten lassen. Dass aber die Berufung auf diesen nationalen Souveränitätsvorbehalt einer besonderen parlamentarischen Legitimation bedürfe, macht angesichts des Kontexts keinen Sinn. Im Gegenteil will das BVerfG ja verhindern, dass dem Bundestag die Regelung elementarer Lebensinhalte entzogen wird. Deshalb ist diese Passage so zu lesen, dass der Verzicht auf das Ziehen der "Notbremse" nur mit Zustimmung des Parlaments möglich ist. Da aber ex ante nie völlig klar sein kann, in welche Richtung sich die Diskussion im Rat entwickeln wird, kann bei jedem strafrechtlichen Rechtsakt prinzipiell die Betätigung der »Notbremse « notwendig werden. Deshalb lässt sich die Forderung des BVerfG am besten erfüllen, wenn bzgl. jedes strafrechtlichen Harmonisierungsakts gem. Art. 82 I, II, 83 I, II AEUV im Bundestag abgestimmt würde. In der Praxis wird die endgültige Fassung eines Rechtsakts allerdings oft erst im Laufe der Konsultationen im Rat ausgehandelt. Damit der deutsche Ratsvertreter in dieser wichtigen Phase ausreichenden Verhandlungsspielraum besitzt, muss eine Lösung gefunden werden, die ihm einen gewissen Freiraum lässt (zB indem das Abstimmungsverhalten unter den Vorbehalt einer nachträglichen Parlamentszustimmung gestellt wird $)^{74}$.

\section{Fazit und Ausblick}

Die vorangegangene Untersuchung hat mehrere »Baustellen« bei der Auslegung strafrechtlicher EU-Kompetenzen nach dem Lissabon-Vertrag aufgezeigt und versucht, hierfür Lösungsansätze $\mathrm{zu}$ präsentieren. Das jüngste Urteil des BVerfG hilft insofern allerdings nur begrenzt weiter. Größere Relevanz entfaltet es für die Begleitgesetzgebung, mit der die Nutzung der »Notbremse" in Art. 82 III und 83 III AEUV geregelt wird. Richtungsweisend kann die Entscheidung für die Entwicklung des Europäischen Strafrechts in erster Linie wegen der darin zum Ausdruck kommenden Grundhaltung sein: Das BVerfG verlangt von allen Akteuren auf europäischer wie nationaler Ebene besondere Sensibilität bei strafrechtlich relevanten Maßnahmen der EU. Diese Mahnung macht es selbst für den Fall interessant, dass die Ratifikation des Lissabon-Vertrags doch noch scheitern und es bei der bisherigen Kompetenzlage bleiben sollte. Das Europäische Strafrecht ist jedenfalls - gerade durch das (erneute) Androhen einer Überprüfung europäischer Rechtsakte durch das BVerfG endgültig in den Mittelpunkt des Interesses gerückt.

68 Für Ausgestaltungsspielräume bzgl. Tatbestand und Rechtsfolgen plädiert auch DANNECKER JURA 2006, 173, 176.

$69 \mathrm{Zu}$ diesem Themenkomplex (und EU-Einflüssen) s. Schönke/Schröder/ Cramer/Heine StGB, 27. Aufl. 2006, Vorbem. $\$ \$ 25$ ff. Rdn. 119 ff.; FROMM ZIS 2007, 279.

70 SATZger IntStR, $\$ 7$ Rdn. 22.

71 Anders noch v. d. Groeben/Schwarze/Prieß/Spitzer EUV/EGV, 6. Aufl. 2004, Art. 280 EGV Rdn. 104 zur alten Rechtslage.

72 Sieber ZStW 121 (2009), 1, 59 hält deshalb nur Richtlinien für zulässig; s. auch SATZGer IntStR, $\$ 7$ Rdn. 42.

73 S. schon Dannecker JURA 2006, 173, 176: »fragwürdige KompetenzKompetenz«.

74 Satzger KritV 2008, 17, 36 f.; skeptisch Schünemann in: ders. (Hrsg.), Gesamtkonzept, 97 (Fn. 15), der auch dann eine »materiell[e] Aushöhlung des Demokratieprinzips im Strafrecht« befürchtet. 